\title{
NUOVE RICERCHE SUL POTENZIALE DI SCARICA NEL CAMPO MAGNETICO.
}

\author{
Memoria del Prof. Sen. AUgUSto Righi ${ }^{1}$ ).
}

\section{Complementi alle esperienze relative alle rarefazioni} medie.

In una precedente pubblicazione ${ }^{2}$ ) ho studiato l'ifluenza del campo magnetico sulla differenza di potenziale necessaria perchè abbia luogo la scarica nell' aria a rarefazione media (pressione di qualche decimo di millimetro), e con elettrodi in forma di dischi paralleli fra loro. I risultati delle mie numerosissime misure furono rappresentati mediante certe curve, aventi per ascisse le intensità del campo magnetico e per ordinate $i$ valori del potenziale di scarica.

Queste curve danno una chiara idea degli effetti dovuti al campo, ed in particolare mostrano in quali circostanze la creazione del campo magnetico faccia comparire la scarica, se prima non esisteva, o la faccia cessare se prima aveva: luogo. Le curve stesse mostrano pure l' esistenza di un campo optimum, cioè di un valore particolare di esso a cui corrisponde il minimo potenziale di scarica; fatto questo non privo d' importanza, e che il sig. Bloch ${ }^{3}$ ) ha pienamente confermato.

Nel citato lavoro mi limitai, oltre che ad un certo ordine di grandezza della rarefazione, anche a valori piccoli della distanza fra un elettrodo e l'altro. Alcune delle nuove

') Estratto dalle Memorie della $R$. Accademia delle Scienze dell' Istituto di Bologna, Serie VI, Tomo VIII, 1910-11.

2) Rend. della R. Acc. di Bologna, 29 maggio 1910.

3) Le Radium, Février 1911, pag. 52. 
esperienze, e precisamente quelle di cui rendo conto in questo primo paragrafo, si riferiscono particolarmente al caso in cui la distanza fra gli elettrodi supera la distanza critica. La disposizione sperimentale addottata è quella stessa delle prime esperienze, salvo qualche modificazione di dettaglio.

Poichè le esperienze precedenti avevano mostrato, che in certi casi il campo determina un aumento del potenziale di scarica anzichè una diminuzione, ho pensato che, se con elettrodi a distanza reciproca maggiore della distanza critica non avevo constatato nessun fenomeno degno di nota, ciò poteva provenire dal fatto, che per le distanze grandi mi ero limitato a ricercare, so il campo magnetico determinasse una diminuzione del potenziale di scarica.

Rimaneva quindi da investigare, se per le grandi distanze fra gli elettrodi si verificasse invece un aumento di potenziale di scarica per opera del magnetismo.

Le esperienze eseguite in proposito hanno dimostrato, che cosi realmente accade.

Ho impiegato all' uopo quello stesso tubo, con elettrodi aventi la forma di dischi paralleli, uno dei quali mobile a piacere, con cui furono fatte le più antiche mie esperienze, ed ho misurato il potenziale di scarica per vari valori della distanza fra gli elettrodi e, per ciascuna distanza, sotto l'azione di campi più o meno intensi; e tutto ciò nei due casi in cui i due dischi sono o perpendicolari o paralleli alla forza magnetica.

La pressione dell' aria nel tubo, che conservo chiuso da tempo, ̀̀ di $0,68 \mathrm{~mm}$.

I seguenti risultati numerici, raccolti con varie serie abbastanza complete di misure, mostrano l'andamento dei fenomeni, che meglio ancora si rileva osservando le fig. 1 e 2.

In queste tabelle le colonne $\mathrm{C}$ contengono $\mathrm{i}$ valori del campo magnetico in gauss; quelle segnate $\mathrm{P}$ danno in volta i valori del potenziale di scarica.

Alcuni schiarimenti sono necessari relativamente al tracciato delle curve. Queste sono distinte in ciascuna delle fig. 1 e 2 colle lettere da $\mathrm{A}$ ad $\mathrm{E}$, corrispondentemente ai cinque 
RICERCHE SUL POTENZIALE DI SCARICA

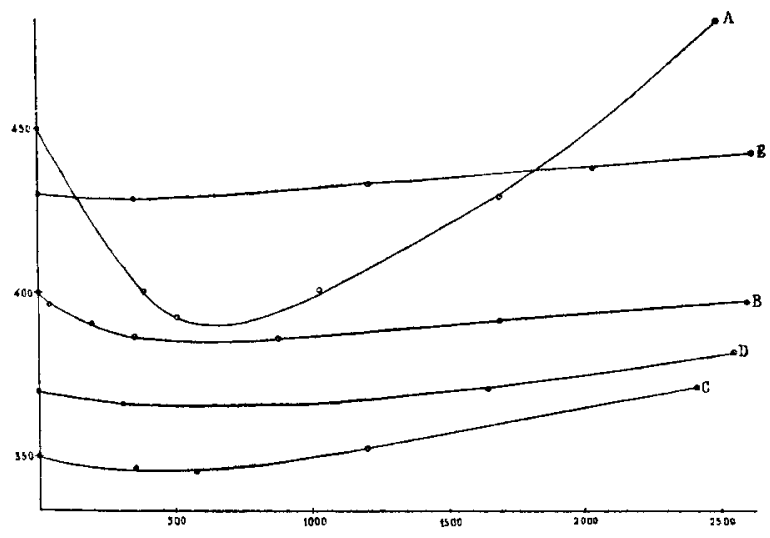

Fig. 1.

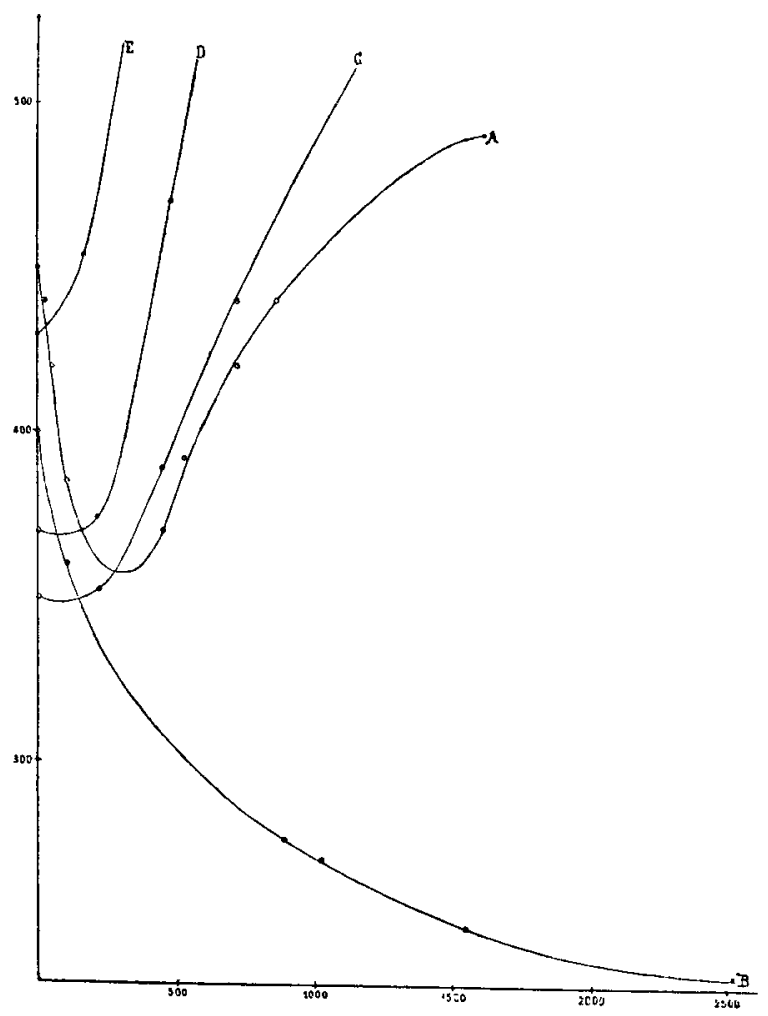

Fig. 2. 
DISCHI PERPENDICOLARI AL CAMPO MAGNETICO.

\begin{tabular}{|c|c|c|c|c|c|c|c|c|c|}
\hline \multicolumn{2}{|c|}{$\begin{array}{l}\text { Dist. in mm. } \\
\text { fra gli elet. } \\
\quad=0,3\end{array}$} & \multicolumn{2}{|c|}{$=1$} & \multicolumn{2}{|c|}{$=8$} & \multicolumn{2}{|c|}{$=10$} & \multicolumn{2}{|c|}{$=15$} \\
\hline $\mathrm{C}$ & $\mathbf{P}$ & C & $\mathbf{P}$ & $\mathrm{C}$ & $\mathbf{P}$ & $\mathbf{C}$ & $\mathbf{P}$ & $\mathrm{C}$ & $\mathrm{P}$ \\
\hline 0 & 450 & 0 & 400 & 0 & 350 & 0 & 370 & 0 & 430 \\
\hline 384 & 400 & 38 & 396 & 346 & 346 & 307 & 366 & 346 & 428 \\
\hline 500 & 392 & 192 & 390 & 576 & 345 & 1640 & 370 & 1200 & 432 \\
\hline 1023 & 400 & 346 & 386 & 1200 & 352 & 25̃35 & 380 & 2010 & 436 \\
\hline 1680 & 428 & 884 & 385 & 2400 & 370 & & & 2585 & 440 \\
\hline 2460 & 480 & 2386 & 390 & & & & & & \\
\hline & & 2586 & 396 & & & & & & \\
\hline
\end{tabular}

DisChI PARALLELI AL CAMPO Magatetico.

\begin{tabular}{|c|c|c|c|c|c|c|c|c|c|}
\hline \multicolumn{2}{|c|}{$\begin{array}{l}\text { Dist. in mm. } \\
\text { fra gli elet. } \\
\quad=0,3\end{array}$} & \multicolumn{2}{|c|}{$=1$} & \multicolumn{2}{|c|}{$=8$} & \multicolumn{2}{|c|}{$=10$} & \multicolumn{2}{|c|}{$=15$} \\
\hline C & $P$ & C & $\mathrm{P}$ & $\mathrm{C}$ & $\mathbf{P}$ & $\mathrm{C}$ & $\mathbf{P}$ & $\mathrm{C}$ & $\mathbf{P}$ \\
\hline 0 & 450 & 0 & 400 & 0 & 350 & 0 & 370 & 0 & 430 \\
\hline 26 & 440 & 102 & 360 & 225 & 352 & 225 & 374 & 153 & 454 \\
\hline 50 & 420 & 883 & 276 & 714 & 440 & 476 & 470 & 422 & 640 \\
\hline 102 & 385 & 1023 & 270 & 1330 & 540 & 861 & 580 & 570 & 740 \\
\hline 450 & 370 & 1545 & 250 & 1730 & 660 & 1253 & 680 & & \\
\hline 524 & 392 & 2440 & 236 & 1972 & 740 & 1545 & 780 & & \\
\hline 714 & 420 & & & & & & & & \\
\hline 861 & 440 & & & & & & & & \\
\hline 1600 & 490 & & & & & & & & \\
\hline
\end{tabular}


valori da $0,3 \mathrm{~mm}$. a $15 \mathrm{~mm}$. dati alla distanza fra gli elettrodi. Le curve $A$ e $B$ si riferiscono dunque a casi di distanze minori della distanza critica, giacchè questa era di circa $6 \mathrm{~mm}$. Sono dunque soltanto le curve $\mathrm{C}, \mathrm{D}, \mathrm{E}$, che corrispondono allo scopo delle esperienze, cui il presente paragrafo è dedicato.

Molte altre serie di misure sono state fatte nelle medesime condizioni con risultati molto concordanti, ed ho tenuto sott' occhio le relative curve quando ho tracciato quelle delle fig. 1 e 2. Ciò mi è stato assai utile, ogni volta che $\mathrm{i}$ punti determinati sperimentalmente riescivano in una data curva troppo lontani l' uno dall' altro.

Dall' esame delle curve C, D, E, si vede subito, come l' effetto dominante del campo $\dot{e}$ un aumento del potenziale di scarica. Tale effetto $\grave{e}$ particolarmente marcato nel caso della fig. 2, ciò̀ nel caso in cui il tubo è collocato in modo, che gli elettrodi siano paralleli alle linee di forza magnetiche.

E evidente la difficoltà che s'incontrerebbe, qualora si volesse render conto in dettaglio di questi fenomeni colla teoria generalmente ammessa.

2. La spiegazione finora ammessa, o la nuova spiegazione proposta.

Si è finora cercato di dar ragione dell' influenza del campo magnetico sul potenziale di scarica ricorrendo al cambiamento di forma delle traiettorie percorse dagli elettroni. Tale spiegazione a me sembra insufficiente per varie ragioni.

In primo luogo, nei casi di debole rarefazione essendo assai piccolo il libero cammino medio degli elettroni, l'azione del campo tendente a modificare il loro movimento fra un urto e l'altro deve verosimilmente produrre effetti poco marcati ; ed in ogni modo non si vede come si possa arrivare a rendere conto degli effetti constatati, e particolarmente delle diminuzioni e degli aumenti del potenziale di scarica, che si manifestano spesso qualunque sia l'inclinazione reciproca fra le linee di forza elettrica e quelle del campo magnetico.

Infine è da osservare, che per render conto dei casi nei quali con una differenza di potenziale inferiore a quella ne- 
cessaria per la scarica, questa si manifesta non appena si crea il campo magnetico, l'ordinaria teoria presuppone che anche prima della creazione del campo esista un passaggio inosservato di elettricità nel tubo di scarica. Ora, non solo ciò non è molto verosimile, ma tutti quelli che hanno voluto mettere in chiaro l' esistenza di tale corrente preesistente all'azione del campo, hanno ottenuto risultati negativi, come rilevai già nel precedente lavoro.

Essendo questo fatto di capitale importanza ho voluto esaminarlo io pure sino dall' inizio delle nuove ricerche, ed ho effettuato per ciò l' esperienza seguente.

Messo uno degli elettrodi del tubo in comunicazione con un sensibilissimo elettrometro a quadranti (e momentaneamente col suolo), ho fatto comunicare l'altro elettrodo attraverso la solita resistenza liquida (destinata ad evitare $\mathrm{i}$ danni di correnti troppo intense) con uno dei poli della batteria di piccoli accumulatori, della quale l'altro polo era in comunicazione col suolo. Togliendo la comunicazione dell' elettrometro colla terra non ho mai osservata la più piccola deviazione, ben inteso essendo il potenziale della batteria minore del potenziale di scarica. Volendo rimanere nel campo dei fatti bisogna quindi convenire, che non $v^{\prime}$ è nel tubo nessun sensibile passaggio di elettricità.

Questa constatazione, da me fatta più e più volte in condizioni svariatissime, e che si accorda con analoghe precedenti osservazioni d'altri fisici, mette nell' alternativa, o di completare, se non abbandonare, l'ordinaria teoria, oppure di ostinarsi ad ammettere un passaggio di elettricità anche prima dell' azione del magnetismo, benchè di tale passaggio non si riesea a dare la dimostrazione sperimentale.

Non bisogna però dimenticare ciò che ho asserito nel precedente lavoro, e ciò che nell' atto in cui il tubo viene incluso nel circuito si ha una corrente di breve durata, che è rivelata dal galvanometro, se questo istrumento $\dot{e}$ inserito in una delle comunicazioni fra il tubo e la batteria, e che ha per effetto, a quanto penso, di produrre un accumulo di ioni positivi presso il catodo e di ioni negativi presso l'anodo. Mentre dunque non esiste ancora una corrente durevole nel 
tubo, ai cui elettrodi è applicata una differenza di potenziale inferiore a quella necessaria per la scarica, il tubo stesso si trova nondimeno in condizioni diverse da quelle in cui si trovava prima d' essere messo in circuito. Tornerò pì̀ avanti su questo fatto, che mi sembra intimamente connesso cogli effetti prodotti dal magnetismo.

Non avendo fede nell' ordinaria teoria ho voluto esaminare se l' ipotesi, già messa avanti nella precedente Nota, secondo la quale il campo magnetico sarebbe in certe circostanze causa di ionizzazione, si prestasse abbastanza bene alla spiegazione dei fenomeni.

Un tale ipotesi non mi sembra priva di fondamento. Infatti, nello stesso modo che il campo magnetico sotto certe condizioni aumenta o diminuisce la stabilità delle coppie neutre elettrone-ione positivo, da me ideate per rendere conto dei fenomeni presentati dai raggi magnetici (o magneto-catodici), esso modificherà in modo analogo le traiettorie percorse dagli elettroni che fanno parte della struttura degli atomi, allargandole se il campo agisce in direzione opportuna. Un esempio gioverà alla chiarezza.

Si consideri in un atomo un elettrone che si mnova circolarmente, e si crei un campo magnetico perpendicolare al piano della traiettoria e diretto in modo, che la forza elettromagnetica agente sull' elettrone in moto risulti diretta dal centro della traiettoria stessa verso l' esterno. Tale forza tenderà evidentemente ad allontanare l' elettrone, e se avrà intensità sufficiente potrà, se non liberarlo, almeno permettergli di separarsi alla più lieve perturbazione esterna. Un campo elettrico, che esista simultaneamente al campo magnetico, potrà agevolare questo risultato, a meno che non si verifichi il caso particolare che la forza elettrica tenda a spingere l'elettrone verso l'atomo.

Non ho affatto la pretesa di ritenere, che i fatti già noti, e quelli che qui andrò descrivendo, forniscano la dimostrazione della verità contenuta in quell' ipotesi; ma credo che essi presentino un soddisfacente accordo colle conseguenze da essa tratte. In ogni modo l' ipotesi stessa è stata per me l' ispiratrice delle nuove ricerche. 
Poichè l' accumularsi di ioni presso gli elettrodi, di eui ho fatto cenno più sopra, crea in vicinanza della loro superficie un campo elettrico, mentre nelle altre regioni del tubo il campo stesso è piccolissimo o nullo, mi sembra rerosimile che la appunto il campo magnetico debba principalmente ma-

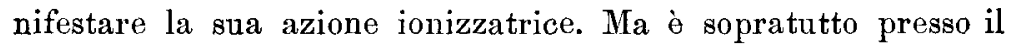
catodo che la supposta magnetoionizzazione tende a provocare la scarica durevole nel tubo. Infatti gli elettroni creati in tal modo, o almeno quelli di essi che sfuggono ad una immediata neutralizzazione per l' incontro d' un ione positivo, acquisteranno presto una velocità considerevole; quelli invece che si creano per magnetoionizzazione presso l'anodo saranno tosto da questo assorbiti, senza cooperare ad iniziare la scarica.

Avendo adottato in via di tentativo questo punto di vista, diveniva necessario assicurarsi anche meglio dell' esistenza di quegli strati di ioni, e rendersi conto per quanto è possibile del modo con cui si producono e degli effetti ai quali possono dar luogo.

3. Corrente o scarica di polarizzazione.

La produzione di ioni, che permangono nel tubo finchè esista fra gli elettrodi una differenza di potenziale minore del potenziale di scarica, fu da me desunta dalla constatazione della corrente di breve durata, che si manifesta nell' atto di stabilire le comunicazioni fra tubo e accumulatori, non che dai caratteri che tale corrente presenta.

Tali caratteri sono i seguenti. Interrotto il circuito per un istante brevissimo e chiudendolo di nuovo quella corrente non si riproduce; ma essa si ottiene nuovamente se la durata dell' interruzione non fu molto breve, e tanto più intensa quanto più durò l' interruzione stessa. Infine, invertendo le comunicazioni fra tubo e sorgente elettrica si ha una corrente assai più intensa che colla semplice chiusura del circuito.

Mi pare, che per spiegare questi fenomeni si debba ragionare nel modo seguente. I pochissimi elettroni liberi eventualmente contenuti nel gas e quelli che possono essere emessi spontaneamente dal catodo iniziano, allorchè il circuito vien 
chiuso, il noto processo della scarica ionizzando per urto le molecole. Quelli fra $i$ ioni positivi in tal modo creati, che non si neutralizzano con elettroni, si portano verso il catodo, mentre gli elettroni, ed $\mathrm{i}$ ioni negativi (prodotti dall' unirsi di elettroni ad atomi neutri) si portano all' anodo. Con ciò il campo elettrico, mentre cresce presso gli elettrodi, diminuisce nel gas e finisce coll' annullarsi, con sospensione d'ogni ulteriore fenomeno, se la differenza di potenziale fra gli elettrodi è abbastanza piccola. Il passaggio della corrente rimane dunque prontamente sospeso.

Le condizioni degli elettrodi del tubo sono allora, sotto un certo aspetto, analoghe a quelle di elettrodi immersi in un elettrolita e polarizzati da una corrente. Bisogna aspettarsi dunque, che il tubo dia una corrente di polarizzazione quando, avendo tolto le comunicazioni fra la batteria d'accumulatori e gli elettrodi, si fanno questi ultimi comunicare con un sensibile galvanometro. Ciò infatti si osserva.

Questa corrente o, forse meglio, scarica di polarizzazione ha una brevissima durata, e la sua intensità integrale, ciò̀ la quantità d' elettricità che la costituisce, dipende naturalmente: $10^{\circ}$ dal tempo $t_{0}$ durante il quale il tubo rimase in comunicazione colla batteria, $2 .^{\circ}$ dal tempo $t$ durante il quale il tubo rimase isolato prima di essere messo in comunicazione col galvanometro.

Conservando a $t$ un valore costante la deviazione galvanometrica è risultata quasi indipendentemente da $t_{0}$.

Per esempio in un caso, por $t_{0}$ eguale a

$$
1-1 \tilde{b}-60
$$

secondi, la deviazione fu rispettivamente:

$$
24-25-25,8
$$

millimetri della scala galvanometrica ${ }^{1}$ ).

Si vede cosi quanto sia rapida la formazione degli strati di ioni sugli elettrodi, e si può praticamente ammettere che ampère.

1) Ogni millimetro corrisponde ad una corrente costante di $4,75 \cdot 10^{-10}$ 
con $t_{0}=30$ secondi la polarizzazione degli elettrodi sia completa.

D'altra parte lo sparire della polarizzazione dopo aver isolati gli elettrodi è invece lenta e graduale, mentre è praticamente istantanea, se si stabilisce una comunicazione metallica fra gli elettrodi.

Per avere una idea della velocità, con cui spontaneamente si attenua ad elettrodi isolati la quantità di ioni accumulati, basta misurare ripetutamente la corrente di polarizzazione, dopo che il tubo è rimasto isolato per un tempo più o meno lungo. Ecco i risultati ottenuti in una fra le molte serio di misure da me eseguite. Adoperai un tubo (che sarà descritto più avanti) avente un catodo costituito da una lamina di alluminio cilindrica applicata contro la parete interna del tubo, ed un anodo cilindrico esso pure e concentrico.

Al tempo $t$ si diedero successivamente $\mathrm{i}$ valori numerici (in secondi) notati nella prima linea della seguente tabella; la seconda linea contiene le corrispondenti deviazioni $d$ ottenute al galvanometro. Il tempo $t_{0}$ fu costantemente $30^{\prime \prime}$.

\begin{tabular}{l|c|c|c|c|c|c|c}
\hline$t$ & 1 & 5 & 10 & 30 & 60 & 120 & 240 \\
\hline$d$ & 52 & 48 & 45,5 & 42 & 38 & 33 & 28
\end{tabular}

La pressione dell' aria nel tubo era un centesimo di millimetro; la batteria dava 2450 volta.

La curva della fig. 3 , che fu disegnata prendendo $t$ come ascissa e $d$ come ordinata, mostra a colpo d'occhio come la provvista di ioni nel tubo diminuisca dapprima rapidamente, poi in modo di più in più lento. Curve di andamento simile ottenni sempre con tubi di forme e in condizioni svariate.

Il graduale distruggersi degli strati di ioni avvolgenti gli elettrodi ha luogo verosimilmente per via di neutralizzazioni reciproche fra essi, e sopratutto fra essi e gili elettrodi. Era dunque da prevedere che un campo magnetico influisso 
su questo fenomeno, per esempio, secondo la teoria ammessa, in causa del mutar di forma delle traiettorie degli elettroni.

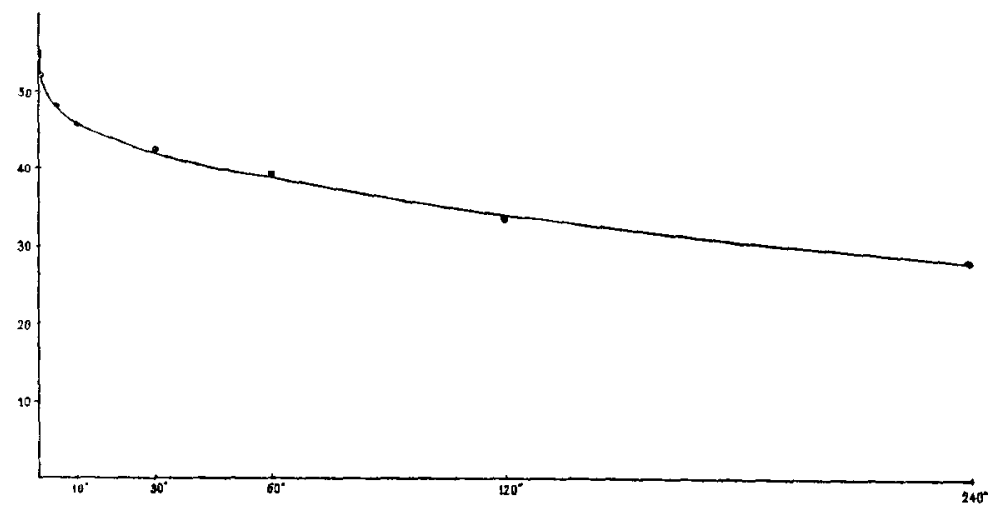

Fig. 3.

Apposite esperienze all' uopo istituite confermano la previsione. Per esse adoperai un inversore a pozzetti di mercurio ben isolati '), col quale era possibile far comunicare i due elettrodi del tubo, ora coi poli degli accumulatori, ora coi serrafili del galvanometro. Generalmente tenevo per mezzo minuto primo il tubo in connessione colla batteria, poi lo isolavo per 10 secondi prima di farlo comunicare collo strumento di misura, il quale colla sua deviazione misurava la quantiti di ioni ancora esistenti. Durante quell' intervallo di 10 , ora ll campo non esisteva, ora invece era in azione. In questo secondo caso ottenni sempre una deviazione minore.

Reco come esempio alcune misure fatte con un túbo cilindrico avente come elettrodi due dischi perpendicolari all'asse del tubo, ed il cui diamatro è poco minore di quello del tubo stesso. La loro distanza è circa 2 centimetri, e l'aria

1) In tutte le esperienze, in cui s' impiegano molti accumulatori e un sensibile galvanometro o elettrometro, è indispensabile il più accurato isolamento di quei conduttori, che non devono comnnicare colla terra. In particolare $\dot{e}$ necessario rivestire a caldo con cera lacca la superficie esterna del tubo all' intorno dei punti da cui escono i conduttori comunicanti coi due elettrodi. 
ha la pressione di circa un cinquantesimo di millimetro. Un campo di 35040 gauss poteva crearsi al momento opportuno.

Orbene, senza campo magnetico la deviazione era 23,9 ; facendo agire il campo, la deviazione era invece 6,9.

Esperienze simili furono fatte successivamente con cinque tubi di forme assai differenti e con svariate pressioni dell' aria interna, ed il risultato, non sempre cosi accentuato come nel caso riferito, fu sostanzialmente il medesimo, e ciò̀ constatai sempre che il campo accelerava la neutralizzazione dei ioni entro il tubo. Anche dopo aver fatto ruotare di $90^{\circ}$ la direzione del campo magnetico constatai la diminuzione della deviazione galvanometrica.

Ammesso ehe un tal risultato sia generale, esso è spesso in disaccordo colla previsione basta sulle deviazioni prodotte dal campo sugli elettroni in moto, giacchè in certi casi quelle deviazioni dovrebbero ostacolare la neutralizzazione delle cariche. Invece si spiega bene l'ascelerarsi della scomparsa dei ioni per opera del campo magnetico, se si ammette la nuova ipotesi proposta, e cioè una ionizzazione prodotta dal campo, particolarmente efficace in prossimità del catodo.

4. Circostanze che influiscono sul potenziale di scarica.

Volendo istituire lo ricerche su tubi a rarefazione piuttosto grande ne ho dapprima adoperato uno della forma ordinaria, ciò̀ munito di elettrodi paralleli di forma circolare e più o meno lontani l'uno dall' altro, collocato fra i poli dell' elettro calamita di Ruhmkorff, ora in modo che $\mathrm{i}$ dischi fossero normali alla direzione del campo, ora disponendoli parallelamente a questa direzione.

Constatai subito che, anche a rarefazioni assai grandi, il campo magnetico in certi casi fa diminuire il potenziale di scarica $\mathrm{e}$ in altri lo fa aumentare; ma pur vincendo lo difficoltà altrevolte segnalate, ebbi grande irregolarità nei risultati numerici. Accadeva poi non di rado ed in modo più pronunciato che colle minori rarefazioni il noto fenomeno, che una volta iniziata la scarica in determinate condizioni, essa perdurava anche se le condizioni stesse venivano poscia modificate in guisa da richierdersi per l'iniziarsi di essa una. 
differenza di potenziale di gran lnnga maggiore di quella effettivamente applicata. Poi mi accorsi, che tale fenomeno si modificava spesso toccando per un momento col dito l'esterno del tubo, o avvicinandovi dei conduttori, o rendendo più o meno buona la proprietà isolatrice della superficie esterna del tubo di vetro. Inoltre constatai, che i fenomeni mutavano alquanto allorchè, pur non facendo variare la differenza di potenziale, si modificava il valore assoluto del potenziale di ciascun elettrodo, per esempio mettendo in comunicazione col suolo ora l' uno ora l'altro dei poli della batteria di accumulatori.

Tutto ciò indicava la formazione di cariche elettriche sulla parete del tubo, le quali cariche subivano naturalmente delle modificazioni per la presenza dei conduttori circostanti. Avendo infatti resa conduttrice la superficie esterna del tubo coll' incollarvi una foglia di stagno, che poi tenni in comanicazione col suolo, le irregolarità e le incertezze sparirono, pur restando l'influenza dei valori assoluti del potenziale. Per studiare questo fenomeno diedi al tubo di scarica la forma indicata dalla fig. 4.

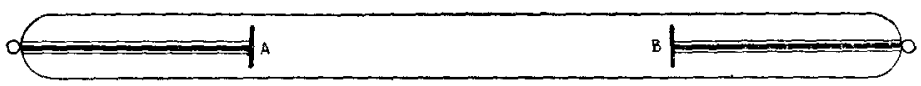

Fig. 4.

Il tubo è cilindrico, e porta come elettrodi A, B due dischetti d'alluminio perpendicolari al suo asse e lontani circa 15 centimetri l' uno dall' altro. Essendo il diametro esterno del tubo poco minore di quello del foro praticato nei nuclei di ferro dei due rocchetti, esso può esservi facilmente introdotto. E siccome l'elettrocalamita è mobile su due guide parallele all' asse dei rocchetti, sj può far in modo, che uno degli elettrodi si trovi in mezzo al campo magnetico, mentre l'altro sta allora entro uno dei rocchetti a metà della sua lunghezza. Con tale disposizione il secondo elettrodo è praticamente sottratto all' azione del campo, almeno finchè la corrente magnetizzante non è molto intensa. 
Per rendermi esatto conto di questa circostanza ho creduto necessario di misurare l' intensità del campo magnetico per varie intensita della corrente magnetizzante, sia nel punto di mezzo della distanza (circa 5 centimetri) fra le faccie polari dei rocchetti, sia a metà lunghezza nell'interno d'ognuno di questi, per mezzo d' un piccolo rocchetto collegato ad un galvanometro balistico. Ecco il risultato di queste misure.

\begin{tabular}{|c|c|c|c|}
\hline \multirow{2}{*}{$\begin{array}{c}\text { intensità } \\
\text { della corrente } \\
\text { in ampère }\end{array}$} & \multicolumn{3}{|c|}{ Campo magnetico in gauss } \\
\hline & $\begin{array}{l}\text { entro } \\
\text { il rocchetto } \\
\text { di sinistra }\end{array}$ & $\begin{array}{l}\text { in mezzo } \\
\text { fra } i \text { poli }\end{array}$ & $\begin{array}{l}\text { entro } \\
\text { il rocehetto } \\
\text { di destra }\end{array}$ \\
\hline 1 & 0 & $3 \check{6} 6$ & 0 \\
\hline 2 & 0 & 1126 & - \\
\hline 4 & - & 1931 & 0 \\
\hline 8 & - & 3018 & - \\
\hline 12 & - & 4064 & 7 \\
\hline 14 & 48 & 4450 & 0 \\
\hline 16 & 113 & 4829 & 88 \\
\hline 20 & 394 & 5473 & 388 \\
\hline 23,5 & 650 & 5955 & 650 \\
\hline
\end{tabular}

La non eguaglianza e l'andamento irregolare dei numeri della $2^{\mathrm{a}}$ e $4^{\mathrm{a}}$ colonna sono verosimilmente doruti ad eterogeneità nel ferro dei due nuclei.

Per le esperienze seguenti l' elettrodo A del tubo si trovava nel mezzo del campo fra i poli, mentre l'elettrodo $B$ si trovava entro il rocchetto di destra. Inoltre per le ragioni dette, il tubo era esternamente rivestito con foglia metallica comunicante col suolo.

Colla disposizione descritta $̀$ facile mettere in evidenza $l$ ' influenza esercitata dal valore assoluto del potenziale dei due elettrodi, come pure l' influenza del segno della loro ca- 
rica, ed ecco una serie di misure, durante le quali la pressione dell' aria nel tubo era di $0,02 \mathrm{~mm}$.

La differenza di potenziale $V$. applicata agli elettrodi, circa 3000 volta, era assai inferiore al potenziale di scarica.

Cambiando le comunicazioni e mettendo a terra uno dei poli della batteria, si potevano realizzare i quattro casi seguenti :

1. Potenziale di $A=+V$; potenziale di $B=0$.

2

2.

$$
\text { " }=0 \quad ; \quad \text { » }
$$$$
\nu=+\mathrm{V} \text {. }
$$

3. $\gg,=-\mathrm{V}$;

$»=0$.

4.

$$
»=0 \quad ; \quad »
$$$$
»=-\mathrm{V} \text {. }
$$

Orbene, mentre perchè si stabilisse nel tubo la corrente (rivelata dalla brusca e permanente deviazione del galvanometro incluso nel circuito) nei casi 2,3 e 4 occorreva un campo magnetico di oltre 5000 gauss, bastava un campo di 1250 nel caso $1^{\prime}$ ).

Lasciando in disparte i casi 2 e 4 , nei quali, per essere a potenziale zero tanto l'elettrodo $A$ che il rivestimento esterno del tubo, il campo elettrico intorno all'elettrodo A non può avere che una intensità debolissima, resta rimarchevole la differenza fra i casi 1 e 3.

Essa può essere attribuita principalmente a queste due circostanze, e ciò $1^{0}$ al fatto che nel caso 1 funziona come catodo la parete intorno ad A, e ciò̀ si ha in certo modo un catodo che avvolge e circonda l'anodo $A$, mentre nel caso 2 avviene l'opposto $; 2^{0}$ ad una diversità di comportamento fra catodi di diverse sostanze.

Contro questa influenza della natura del corpo, su cui vanno a terminare le linee di forza elettrica, parla l' esperienza seguente. Un tubo simile a quello della fig. 4 , il quale però in luogo del rivestimento metallico esterno ne ha uno interno, costituito da una laminetta d'alluminio piegata a cilindro, applicata contro la parete del tubo e comunicante col suolo, dà sostanzialmente gli stessi risultati or ora descritti.

1) Questa ed alcune delle esperienze che deseriverò piư oltre furono gia concisamente descritte altrove (Comp. Rend. 30 Janvien 1911). 
Tuttavia, non parendomi da escludere a priori una qualche influenza della natura del catodo, o generalmente dei corpi che, ricevendo linee di forza, possono come tali comportarsi entro un tubo di scarica, ho creduto bene istituire ricerche speciali col tubo, di cui la fig. 5 mostra la sezione.

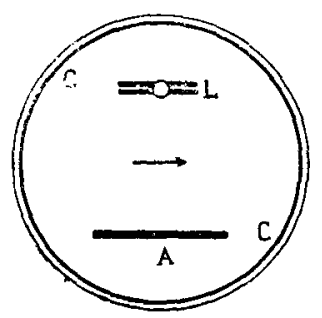

Fig. 5.

trasversale. In qnesto tubo (diametro circa 4 cent.) il catodo ò costituito da una lamina cilindrica di alluminio C applicata contro la sua parete interna, mentre l'anodo è una lastra rettangolare A (3 c. per 1,7 c.). Questa lastra è eccentrica, onde lasciar posto ad un'altra L ad essa parallela lunga 5 c. e larga 1,2 c. costituita da due lamine sovrapposte, una d'alluminio e l'altra di diversa sostanza p. es. di piombo. Questa doppia lamina $L$ può girare intorno ad un asse parallelo all' asse del tubo, ed essere disposta in modo da presentare all' anodo A ora la faccia di alluminio ora quella di piombo, essendo a tal uopo fissata ad un giunto smerigliato.

Nell' esperienza eseguita l' aria nel tubo aveva la pressione di $0,012 \mathrm{~mm}$., la batteria forniva agli elettrodi una differenza di potenziale di 1730 volta, e al momento opportuno si creava un campo di circa 2500 gauss diretto come la freccia della fig. 5. Ecco ciò che ho osservato.

Mentre senza campo la corrente non si stabiliva nel tubo, la corrente stessa non si iniziava neppure col campo, se verso l' anodo era rivolta la faccia d' alluminio della doppia lamina L. Ma se verso l' anodo era rivolto il piombo la corrente si produceva. Lasciando poi sussistere il campo e facendo girare la doppia lamina in modo continuo, la corrente cessava ogni volta che all' anodo si volgeva la faccia d'allu- 
minio, per ristabilirsi quando invece era il piombo che riceveva dall' anodo delle linee di forza elettrica.

Col platino al posto del piombo ho avuto un risultato analogo, ma assai meno pronunnciato, mentre che con vetro, argento, rame, ottone, zinco e bismuto non ho ottenuto risultati sicuri.

Mentre dunque la differenza fra $i$ casi 1 e 3 precedenti

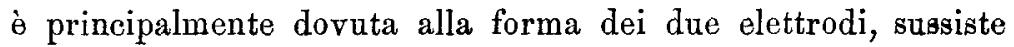
certamente almeno pel piombo una certa attitudine a favorire la scarica, quando su di esso vanno a terminare delle linee di forza elettrica. Il fatto potrebbe ascriversi a traccie di radioattività, o a maggior tendenza ad emettere elettroni sotto I' azione della forza elettrica. In ogni modo si tratta d'un fenomeno probabilmente estraneo a quelli studiati nel presente lavoro.

Si potrebbe ancora pensare, che il modo leggermente diverso di comportarsi fra catodi di diversa natura fosse legato ad una diversa facilità di lasciare uscire i gas occlusi. Questa idea, che forse meriterebbe qualche considerazione, ̀̀ suggerita dal fatto seguente da me molte volte constatato. Se si determina il valore del potenziale di scarica per un dato tubo, e poi coi noti metodi (ulteriore rarefazione accompagnata dal passaggio d' una corrente nel tubo) si cerca di sottrarre agli elettrodi i gas aderenti o occlusi, e, ridotta la prossione al valor primitivo si rinnova la misura, si trova sempre un notevole aumento del potenziale di scarica.

Dalle descritte esperienze si trae l'impressione che, se davvero il campo magnetico può ionizzare il gas, ciò principalmente avviene presso il catodo, ciò che si accorda con quanto fu detto nel $\S 2$. Però non resta escluso, che la magnetoionizzazione si produca anche altrove, ed $\dot{e}$ anzi per chiarire questo punto che furono istituite esperienze, che saranno descritte più avanti.

5. Misure del potenziale di scarica nel campo magnetico in casi di grande rarefazione.

Visti gl' inconvenienti prodotti dal vetro delle pareti del tubo, ho dovuto adottare, per eseguire una serie sistematica 
di misure, dei tubi, nei quali uno degli elettrodi è costituito da una lamina metallica applicata alla parete. La fig. 6 mostra un simile tubo, col quale ho potuto fare molte e concordanti determinazioni.

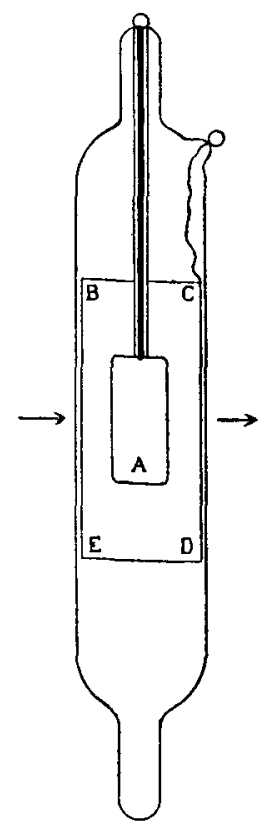

Fig. 6.

Mentre uno degli elettrodi è costituito dalla lamina cilindrica BCDE (altezza del cilindro 10 cent. diametro 3,5), l'altro è una lamina piana A (3 cent. per 1,7). Il tubo può essere girato intorno al proprio asse, e quindi essere collocato in guisa, che l'elettrodo $A$ sia o parallelo o perpendicolare alla direzione, indicata dalle freccie nella figura, del campo magnetico.

In causa della differenza di forma fra i due elettrodi, e della circostanza che l' elettrodo A non ha forma di rivoluzione coassiale al tubo, si dovranno esaminare quattro casi diversi. Infatti, per ciascuna delle sue orientazioni principali della lastra $A$, questa può essere anodo o catodo. 
L' effetto prodotto dal campo magnetico è differente nei quattro casi. Si hanno ciò̀ quattro diverse curve rappresentatrici del fenomeno, costruite nel solito modo, ciò̀ prendendo per ascissa il campo e per ordinata il corrispondente potenziale di scarica. In un gruppo di misure coll' aria a 0,056 mm. di pressione ho raccolto i dati della tabella seguente.

\begin{tabular}{|c|c|c|c|c|c|c|c|}
\hline \multicolumn{4}{|c|}{ Lastra $\mathbf{A}+$} & \multicolumn{4}{|c|}{ Lastra A- } \\
\hline \multicolumn{2}{|c|}{ ierpendic. al campo } & \multicolumn{2}{|c|}{ parallela al campo } & \multicolumn{2}{|c|}{ perpendic. al campo } & \multicolumn{2}{|c|}{ parallela al campo } \\
\hline $\mathbf{P}$ & $\mathbf{C}$ & $\mathbf{P}$ & $\mathrm{C}$ & $\mathbf{P}$ & $\mathrm{C}$ & $\boldsymbol{F}$ & $\mathrm{C}$ \\
\hline \multirow{3}{*}{300} & ( 153 & 400 & 422 & \multirow{3}{*}{380} & 76 & 580 & 一 \\
\hline & 430 & \multirow[b]{2}{*}{450} & 383 & & 254 & 630 & 0 \\
\hline & 127 & & $\{4500$ & & $\mathbf{3 8}$ & 640 & 137 \\
\hline \multirow[t]{2}{*}{400} & $\{603$ & & $\int 229$ & 500 & 317 & 690 & 1593 \\
\hline & 76 & อ๊อ๊0 & 1370 & \multirow{2}{*}{550} & 13 & 695 & 2474 \\
\hline \multirow[t]{2}{*}{610} & 1447 & & $\langle 216$ & & 383 & 700 & 5473 \\
\hline & $\int 61$ & o90 & 1880 & 620 & 396 & \multicolumn{2}{|c|}{$\mathrm{D}$} \\
\hline \multirow{2}{*}{650} & 1750 & & ( 152 & 850 & 643 & & \\
\hline & 661 & 660 & $\{2535$ & 10500 & 947 & & \\
\hline \multirow{2}{*}{700} & $\{\ldots$ & 750 & 127 & 1410 & 1690 & & \\
\hline & $\{13$ & $84 \overline{0}$ & 102 & 1720 & 3307 & & \\
\hline \multirow[t]{2}{*}{840} & 3175 & 930 & 77 & 1830 & 5196 & & \\
\hline & & & & & & & \\
\hline
\end{tabular}

La disposizione dei numeri in questa tabella è differente da quella delle tabelle riportate nel $\$ 1$, e cioè proviene dall'avere alcun poco modificato il metodo delle misure. Anzichè, come in passato, dare al campo magnetico un determinato valore e poi aumentare d'una unità per volta il numero degli accumulatori sino ad avere la scarica, mi sono persuaso che vi è vantaggio a fare l' inverso, e ciò̀ dare un 
determinato valore al potenziale fornito dalla batteria, e poi far variare lentamente l'intensità del campo magnetico (per mezzo di reostati a corsoio inseriti nel circuito della corrente magnetizzante) sinchè la scarica si produca ${ }^{1}$ ).

Così nel caso della prima delle misure registrate in detta tabella, dopo aver dato alla differenza di potenziale il valore di 300 volta, feci crescere il campo gradatamente a partire da zero sinchè, arrivato a 153 gauss, la corrente bruscamente si produsse.

Siccome però in molti casi ad uno stesso valore del potenziale di scarica possono corrispondere valori diversi (due e qualche volta tre) del campo magnetico cosi, dopo quella prima determinazione, occorreva cercare se esistevano altri valori del campo. Perciò diedi a questo un alto valore, per esempio 2000 gauss, e visto che la corrente non passava, lo feci gradatamente diminuire. Arrivando a 430 gauss la corrente di nuovo si produsse. $\mathrm{E}$ siccome da 2000 gauss in più non si ebbe corrente, cosi conclusi, che a 330 volta corrispodono i valori 153 e 430 gauss del campo, come pure che v'è corrente nel tubo solo se il campo magnetico ha un valore compreso fra questi due limiti.

Se per un certo valore del campo maggiore di 430 gauss si fosse nuovamente stabilito il passaggio della corrente nel tubo, avrei dovuto registrare nel quadro questo terzo valore. $\grave{\mathrm{E}}$ assai probabile che in qualche caso il non aver trovato un terzo valore si debba a ciò, che per trovarlo sarebbe stato necessario realizzare campi di molto grande intensità.

Alle quattro serie di misure della precedente tabella, distinte colle lettere $\mathrm{A}, \mathrm{B}, \mathrm{C}, \mathrm{D}$, corrispondono ordinatamente le curve segnate colle stesse lettere nella fig. 7 .

L' andamento delle tre prime somiglia assai a quello di molte delle curve del caso di media rarefazione, mentre l'andamento della $\mathrm{D}$ (catodo piano perpendicolare al campo) è assai differente, in quanto che con potenziali minori del poten-

1) Ho trascurato sempre le scariche momentanee, che qualche volta precedono lo stabilirsi della scarica permanente. 
ziale di scarica ordinario (ciò̀ senza campo) il campo magnetico non produsse effetto sensibile, e fu necessario ricorrere a

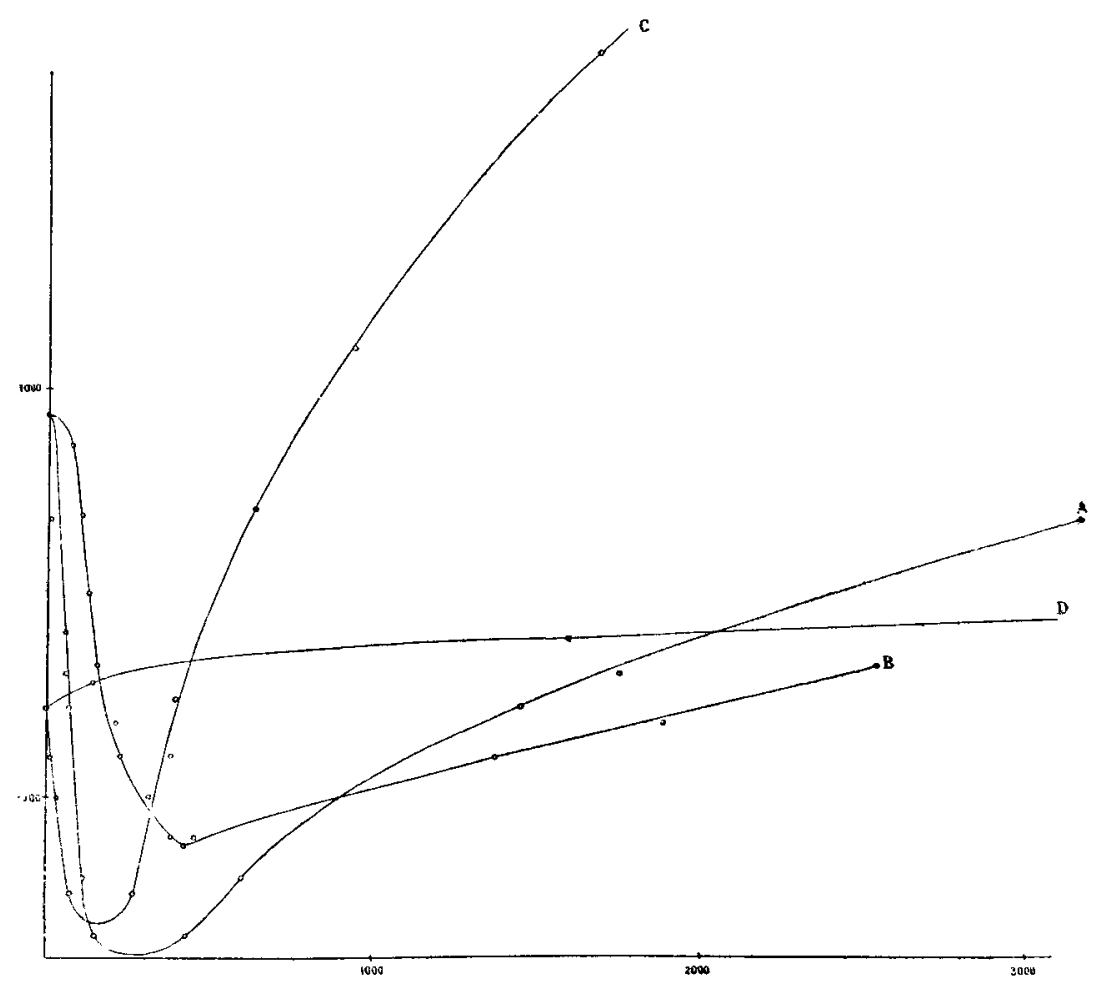

Fig. 7.

potenziali più elevati per constatare un effetto, e precisamente riconoscere che il campo fa aumentare il potenziale necessario alla produzione della corrente.

6. Esperienze con tubi aventi elettrodi di forme varie. Sia secondo l'ordinaria spriegazione, sia secondo la nuova, che serve a completare la prima, la forma degli elettrodi deve esercitare una considerevole influenza sull' andamento dei fenomeni.

Infatti, è in tubi diversi necessariamente differente la distribuzione e forma delle linee di forza come pure l'inten- 
sità del campo elettrico presso $i$ vari punti della superficie del catodo. Non era dunque senza interesse l' istituire misure analoghe a quelle del paragrafo precedente, con tubi aventi elettrodi di svariate forme e variamenti disposti. Mi limiterò quì a recare tre esempi.

a) Il tubo di scarica ̀̀ rappresentato dalla fig. 8, e contiene aria a $0,03 \mathrm{~mm}$. di pressione. Uno degli elettrodi B consiste in un tubo d'alluminio applicato contro la parete,

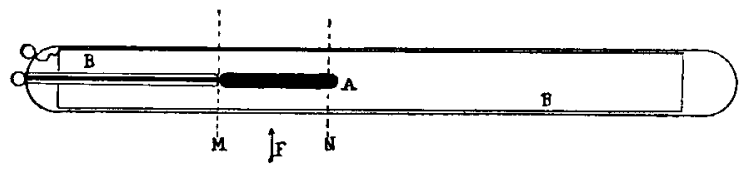

Fig. 8

mentre l'altro $\mathrm{A}$ è un cilindro dello stesso metallo lungo $\check{\sigma}$ centimetri circa e grosso mezzo centimetro. Nelle due prime serie di misure il tubo fu introdotto nei nuclei dell'elettrocalamita, in modo che il campo magnetico, per tal modo diretto nel senso del suo asse, agisce quasi esclusivamente sulla porzione del tubo corrispondente all' elettrodo A. Le faccie polari dell'elettrocalamita arrivavano infatti in $\mathrm{M}$ ed N. Nelle altre due serie di misure il tubo era collocato trasversalmente al campo, e cioè questo aveva la direzione della freccia $\mathrm{F}$.

La tabella seguente porge i risultati numerici ottenuti, coi quali ho poi disegnate le curve della fig. 9 ').

1) Per economia di spazio non compaiono nella figura quelle porzioni di curva che corrispondono ai più grandi valori del campo e del potenziale. 


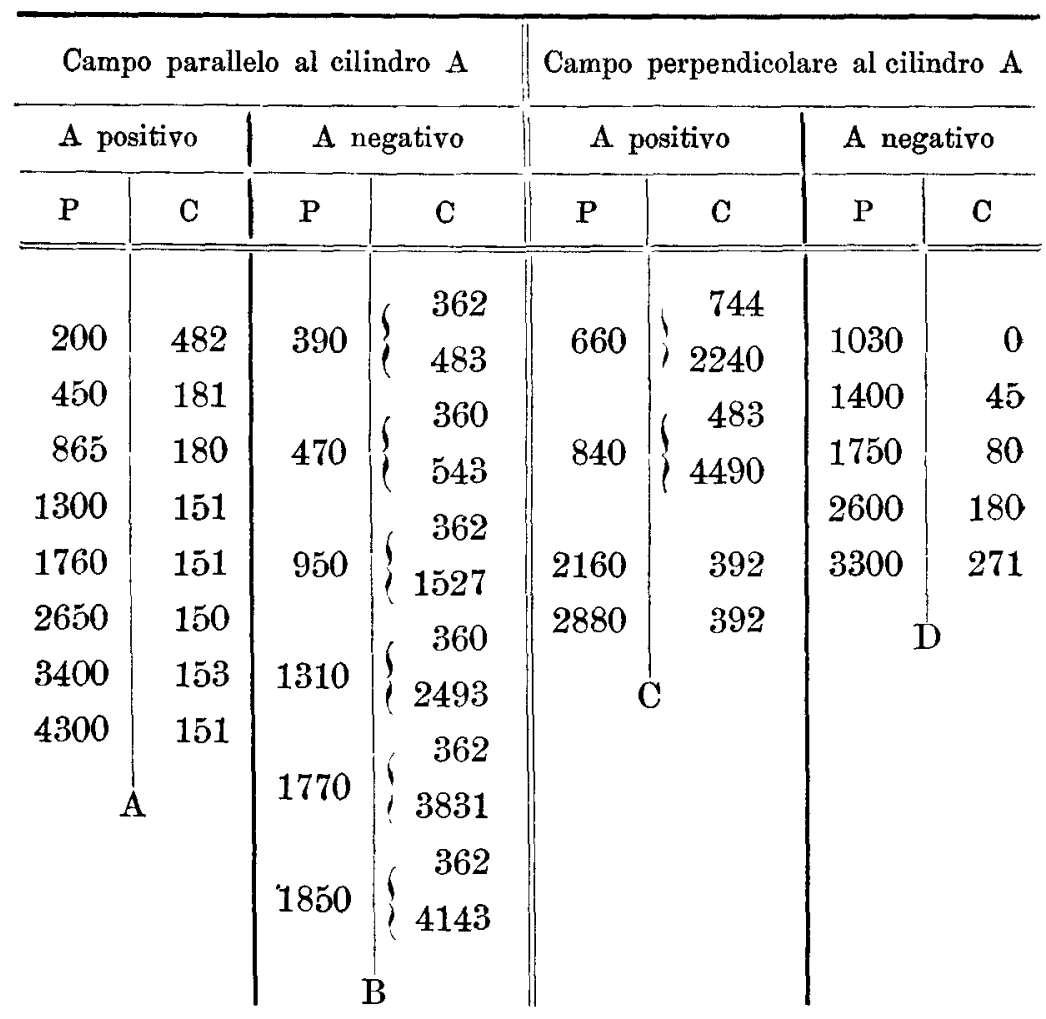

Per distinguere le curve della fig. 9 ho segnato presso di esse le stesse lettere, che nella precedente tabella designano le varie serie di misnre.

Avrò occasione più avanti di mostrare la curva $B$ ottenuta collo stesso tubo con pressione interna più elevata $\mathbf{e}$ allora si vedrà che la curva stessa, dopo la grande salita di destra, raggiunge una massima altezza per poi ridiscendere. Colla rarefazione più spinta per giungere fin là sarebbe stato necessario creare campi magnetici assai più intensi di quelli disponibili.

b) Un secondo esempio l'offre il tubo da scariche rappresentato in sezione dalla fig. 10. Entro il tubo di vetro sono applicate contro le pareti quattro eguali lastre d' alluminio, le quali, salvo il piccolo intervallo che le separa nel 
senso delle generatrici, costituiscono col loro insieme un cilindro. Due di esse A, A, fra loro comunicanti, costituiscono

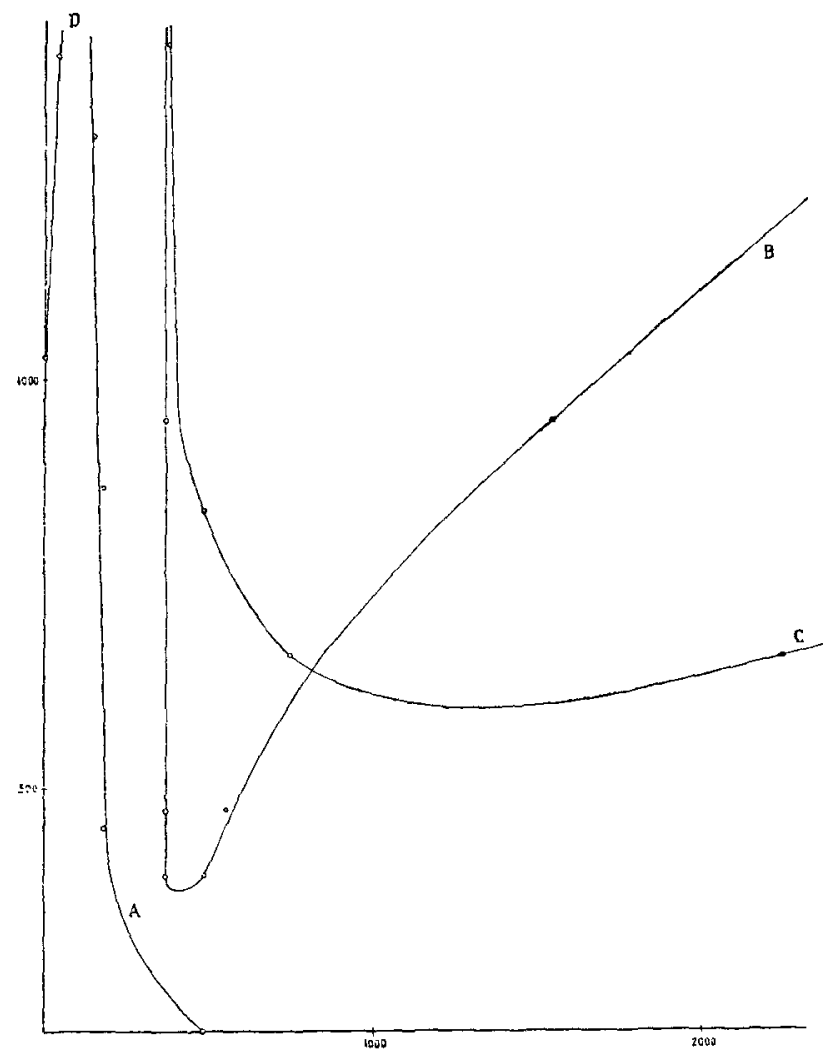

Fig. 9.

uno degli elettrodi, le altre due B, B, costituiscono l'altro. Si hanno dunque due elettrodi di forma identica, senza, tutta-

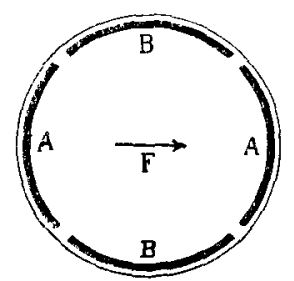

Fig. 10. 
via, che resti scoperta la parete di vetro (salvo le strette strisce fra una lastra e l'altra). Il campo magnetico agisce nella direzione della freccia $\mathrm{F}$, ciò̀ secondo la retta che congiunge $\mathrm{i}$ centri delle due lastrine costituenti uno degli elettrodi A A. Questo elettrodo era anodo nella prima serie dello misure seguenti, e catodo nella seconda. La pressione nel tubo era $0,04 \mathrm{~mm}$.

\begin{tabular}{|c|c|c|c|}
\hline \multicolumn{2}{|c|}{ Elettrodo A A + } & \multicolumn{2}{|c|}{ Elettrodo A A - } \\
\hline$P$ & C & $P$ & $\mathrm{C}$ \\
\hline & & & 229 \\
\hline 1570 & 0 & 350 & 330 \\
\hline 1620 & 25 & & 160 \\
\hline 1830 & 102 & 415 & 632 \\
\hline 1980 & 153 & & 127 \\
\hline 2190 & 343 & 530 & 1992 \\
\hline 2370 & 512 & & 120 \\
\hline 2450 & $59 \overline{0}$ & 590 & 5674 \\
\hline 2830 & 6240 & & \\
\hline & & 740 & 114 \\
\hline & & 930 & 102 \\
\hline & & 1040 & 102 \\
\hline
\end{tabular}

Le curve della fig. 11 sono disegnate coi precedenti numeri, ed i segni + , 一, servono a distinguerle. Per non dare alla figura dimensioni soverchie, non solo si sono soppresse le porzioni relative ai più elevati valori del campo, ma si sono disegnate le due curve una dentro l'altra in grazia d'un opportuno spostamento degli assi di riferimento. E degna di nota la grande diversità fra le due curve, diversità che in 
questo caso speciale è quella che, in parte almeno, si accorda coll' ordinaria spiegazione dei fenomeni.

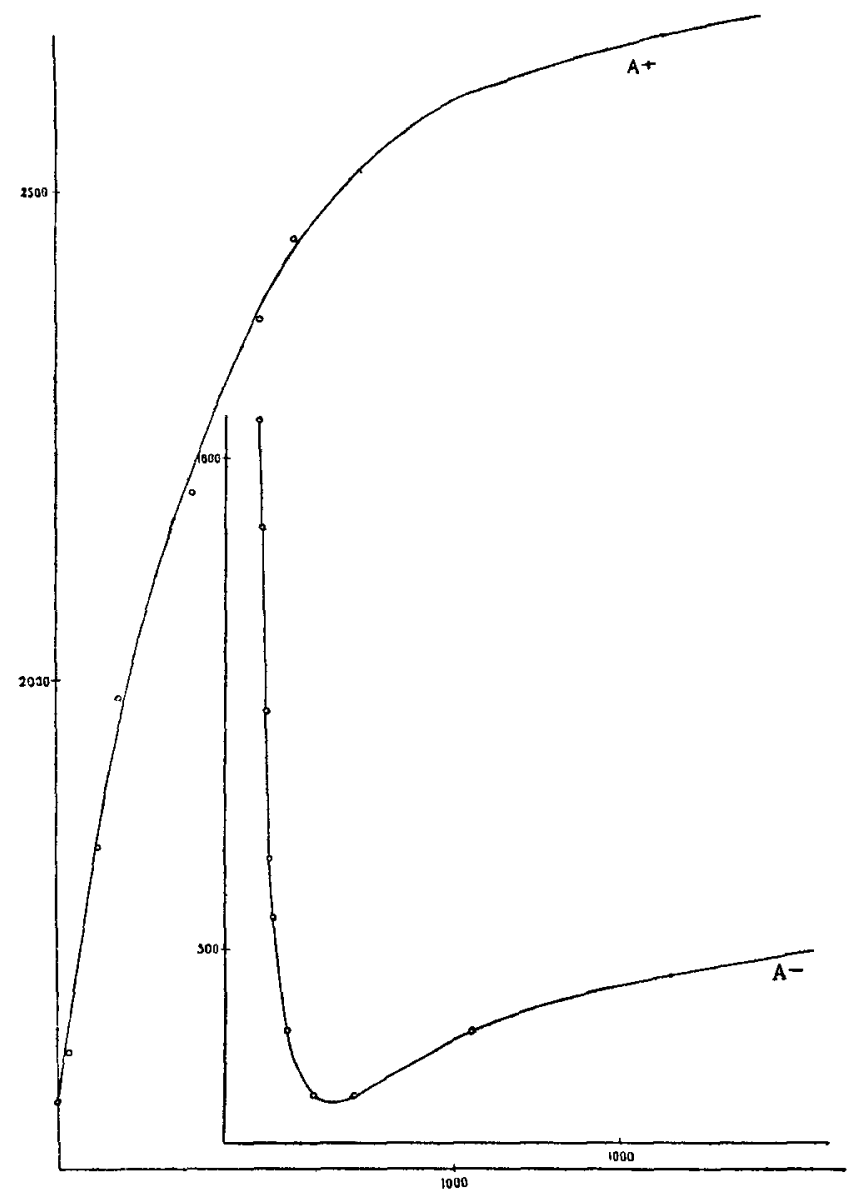

Fig. 11.

I due esempi precedenti $a$ ), b) mettono in evidenza la varietà dei fenomeni, ma anche qualche carattere costante, comune cioè ai vari tubi. Non sarebbe facile servirsi dei dati precedenti a scopo di verifica qualitativa d' una qualunque delle spiegazioni, in causa delle forme speciali degli elettrodi. 
Ad uno scopo di questo genere si presterebbe meglio il terzo esempio che segue.

c) Il tubo adoperato differisce da quello della fig. 8 soltanto in ciò, che l'elettrodo A ̀̀ lungo quanto il tubo. Introdotto quest' ultimo nei rocchetti, le condizioni dell' esperienza sono sensibilmente quelle di un c ampo elettrico cilindrico a d'un campo magnetico uniforme, le cui linee di forza sono perpendicolari alle linee di forza elettrica; ed in questo caso speciale è possibile determinare la forma delle traiettorie percorse dagli elettroni emessi dal catodo.

Mi limiterò al caso in cui il cilindro centrale è catodo; ma darò $i$ risultati ottenuti con due differenti gradi di rarefazione dell' aria entro il tubo.

\begin{tabular}{|c|c|c|c|}
\hline \multicolumn{2}{|c|}{ Pressione $=0,012$} & \multicolumn{2}{|c|}{ Pressione $=0,1$} \\
\hline $\mathrm{P}$ & $\mathrm{C}$ & $P$ & $\mathrm{C}$ \\
\hline 750 & 5141 & & 259 \\
\hline \multirow[t]{2}{*}{2100} & ว2231 & 190 & 405 \\
\hline & 563 & & 5200 \\
\hline \multirow[t]{3}{*}{2860} & 1126 & & 236 \\
\hline & 5392 & 309 & 900 \\
\hline & 563 & & 5151 \\
\hline \multirow[t]{3}{*}{2950} & 1528 & & 225 \\
\hline & 5310 & 940 & 2800 \\
\hline & 475 & & 4919 \\
\hline \multirow[t]{3}{*}{3800} & $\overline{2} 800$ & & 220 \\
\hline & วั419 & 1480 & $4 \check{5} 05$ \\
\hline & & & 4849 \\
\hline
\end{tabular}

La fig. 12 mostra la curva costruita coi numeri relativi alla pressione 0,1 . Per la pressione 0,012 si avrebbe una 
curva simile a quella della fig. 12 , ma limitata ai più bassi potenziali. La curva sarebbe quindi costituita da due tratti separati fra loro.

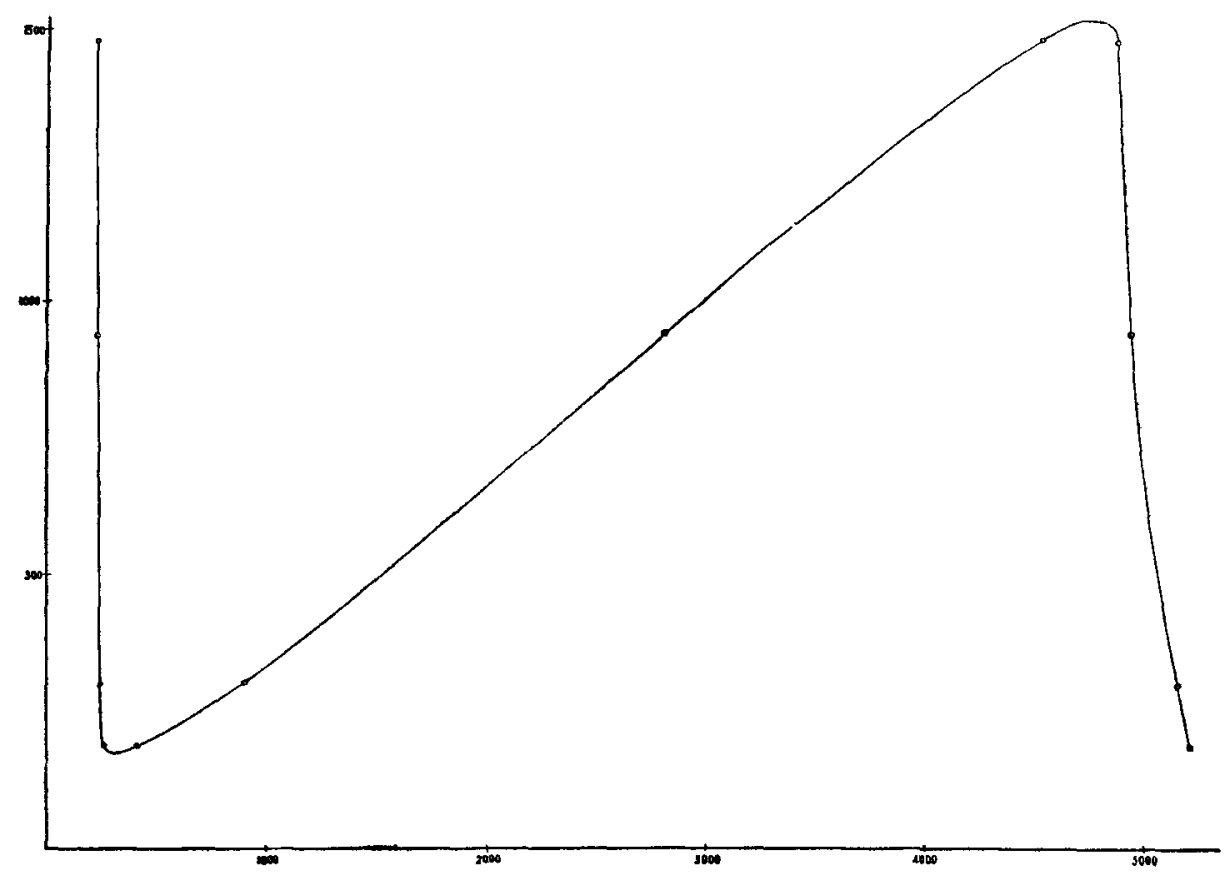

Fig. 12.

La curva fig. 12 sembra essere il tipo più generale rappresentante la relazione fra intensità di campo magnetico e potenziale di scarica, almeuo per i tubi il cui anodo è cilindrico e circonda il catodo ${ }^{1}$ ). Si notano in essa due tratti poco differenti da rette verticali. Quello relativo a campi di 200 a 300 gauss ha però spesso una tale forma (per es. nella fig. 14) da dimostrare, che ad uu medesimo campo cor-

1) Probabilmente se si disponesse di campi assai più intensi di 5000 gauss, e si potesse cosi ulteriormente prolungare la curva, questa, dopo aver raggiunto una nuova ordinata minima, diverrebbe ancora ascendente. La curva avrebbe allora all' incirca la forma della lettera $M$ capovolta. 
rispondono due diversi valori del potenziale di scarica; ma probabilmente ciò si deve a qualche inavvertito errore sistematico di poca entità. In rari casi ciò si è verificato anche per il secondo tratto di discesa della curva, che corrisponde a campi assai intensi.

Quei tratti di curva quasi verticali rappresentano brusche diminuzioni del potenziale di scarica dovute verosimilmente a due cause distinte, che l'ipotesi della magnetoionizzazione spiega agevolmente. $\mathrm{E}$ cioè, la prima ripida discesa della curva deve attribuirsi all' azione del campo sul catodo; la seconda invece alla azione sul gas lontano da questo elettrodo.

\section{zazione. \\ 7. Esperienze suggerite dalle ipotesi della magnetoioniz-}

Facilmente s'intuisce la maniera con cui si può sottoporre la precedente interpretazione alla prova dell'esperienza. Basterà costruire la curva caratteristica per un tubo del tipo fig. 8 due volte, e cioè quando l'elettrodo $\mathrm{A}$, adoperato come catodo, si trova fra i poli della elettrocalamita e poi, dopo avere convenientemente spostata questa, quando il catodo si trova entro uno dei rocchetti, ove $\dot{e}$ sensibilmeute sottratto all' azione del campo magnetico.

La prima delle due curve fn già ottenuta, ed è la curva $\mathrm{B}$ della fig. 9. Ma essa non è completa, mancando della soconda ripida discesa, pel motivo che nelle misure non si raggiunsero valori abbastanza grandi del campo magnetico. Ho dovuto quindi rifarla anch' essa.

Prima però d'esporre i risultati delle nuove misure è bene eliminare un dubbio, che sorge spontaneo nella mente, e cioè è utile anzitutto verificare, che il campo non dia il suo effetto sul catodo, anche quando questo si trova entro uno dei rocchetti.

A questo scopo ho costruito il tubo rappresentato dalla fig. 13, che differisce da quello della fig. 8 in ciò, che il suo catodo $A$ ha la forma d' un disco perpendicolare all' asse del tubo. Inoltro all' esterno e in corrispondenza del catodo formai un piccolo rocchetto $R, R$, avvolgendo sul vetro per dieci 
giri un sottile filo di rame ben isolato, i cui capi, torti strettamente assieme, furono poi messi in comunicazione con un sensibile galvanometro balistico. Leggendo la deviazione prodotta, quando s' interrompe e si chiude una corrente di nota intensità nei rocchetti, si può cosi calcolare facilmente l'intensità del campo nel luogo occupato dal catodo.

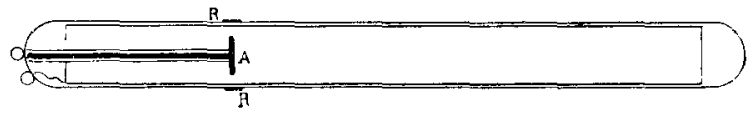

Fig. 13.

Ecco i risultati d' una esperienza, fra le molte concordanti da me esegnite, mentre l'aria entro il tubo aveva 0,028 $\mathrm{mm}$. di pressione.

Disposto il tubo entro i rocchetti e collocata l' elettrocalamita in modo che il catodo A fosse fra i poli a meta distanza, trovai, che con una differenza di potenziale di 2210 volta applicata agli elettrodi occorreva un campo di almeno 3713 gauss, perchè si determinasse il passaggio della corrente.

Spostata allora l' elettrocalamita sinchè il catodo venisse a trovarsi entro uno dei rocchetti a metà della lunghezza di questo, fu necessario portare il campo a 4829 gauss per avere ancora la scarica. Per decidere se in questo caso si trattava d' un' azione sull' aria collocata fra i poli, o di una azione sul catodo, era necessario conoscere a quale intensità di campo si trovava esposto quest' ultimo.

Col piccolo rocchetto trovai, che mentre il campo aveva fra i due poli l' intensità di 4829 gauss, l' intensità intorno al catodo era soltanto 276 gauss, cioè circa la quindicesima parte del valore (3713) necessario a determinare la scarica. Questa non poteva ascriversi dunque all' azione del campo magnetico sul catodo collocato entro il rocchetto.

Con altri gradi di rarefazione, o con altri valori del potenziale applicato al tubo, ottenni sempre un analogo risultato, in modo più o meno marcato a seconda dei casi. L' esempio precedente corrisponde a quella fra le varie espe- 
rienze in cui il fatto si manifestava nel modo più accentuato.

Fatta questa constatazione, che da sola rende gia oltremodo probabile la magnetoionizzazione dell' aria lontana dal catodo, ho eseguite le misure necessarie per costruire le due curve, di cui ho parlato più sopra. Ho adoperato il tubo della fig. 8, con aria alla pressione di 0,088 mm. Eicco i risultati ottenuti.

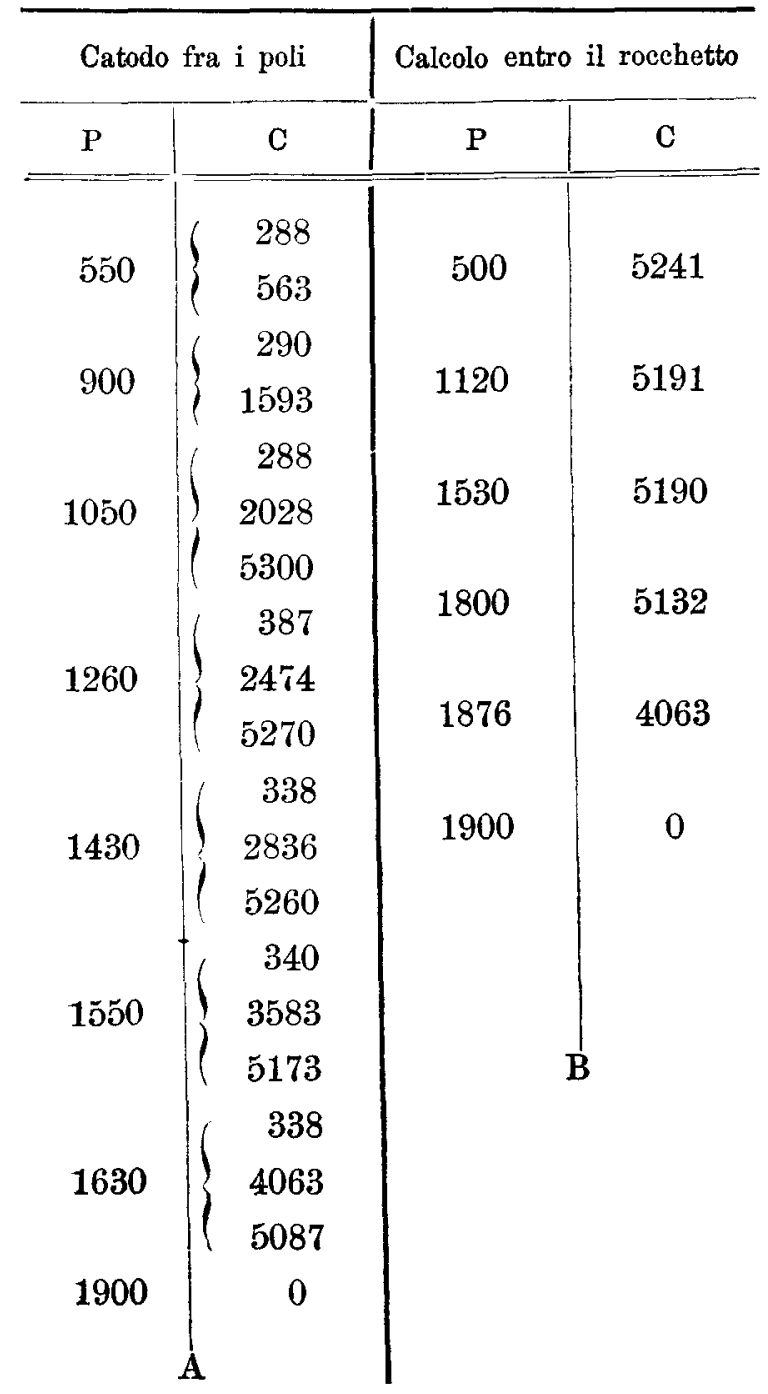


Le curve A, B della figura 14, costruite coi numeri precedenti, mostrano l' andamento dei fenomeni, e dal loro confronto si rileva subito, che col sottrarre il catodo all' azione del campo si sopprime la parte in ripida discesa corrispondente ai valori del campo intorno a 300 gauss, mentre resta la seconda parte discendente relativa ai campi di circa 5000 gauss. Da ciò mi sembra risultare una buona conferma delle previsioni basate sulla ipotesi della magnetoionizzazione.

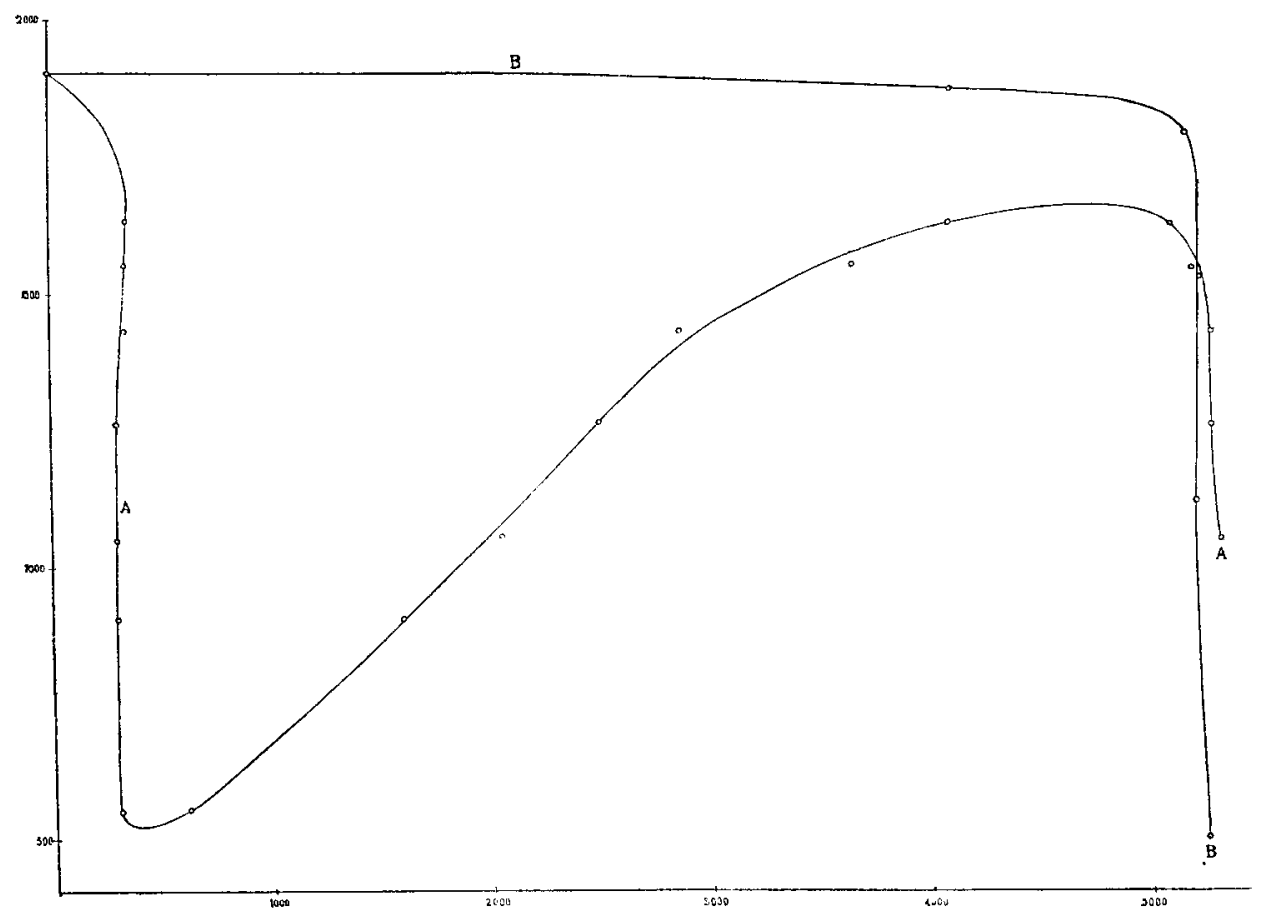

Fig. 14.

Non sarà superfluo descrivere un'altra esperienza, che in fondo non è che un variante delle precedenti.

Mi sono servito di un tubo simile a quello della fig. 4, ma senza il rivestimento esterno di stagnuola (che, come ho potuto verificare, non modificherebbe sostanzialmente il risultato), il quale tubo ha i suoi elettrodi cosi lontani l'uno dal- 
l'altro, ehe, quando uno si trova entro il nucleo d' un rocchetto a metà della lunghezza di questo, l' altro va ad occupare la posizione simmetrica entro l'altro rocchetto. Durante le misure il punto di mezzo della batteria veniva messo in comunicazione colla terra, in modo che i potenziali dei due elettrodi avessero valori eguali e di segno contrario. Ciò tendeva a rendere perfettamente longitudinale il campo elettrico nella parte del tubo, la più Iontana dai due elettrodi, che rimaneva nell' intervallo fra i poli magnetici. Con pressione di $0,04 \mathrm{~mm}$. nel tubo ebbi questi risultati:

\begin{tabular}{c|c}
\hline $\mathrm{P}$ & $\mathrm{C}$ \\
\hline 450 & 5090 \\
760 & 4910 \\
920 & 4678 \\
1082 & 4510 \\
1210 & 4140 \\
1220 & 0
\end{tabular}

Non riprodurrò la curva che ne ritulta, perchè essa ̀̀ assai simile alla B della fig. 14, salvo che la parte discendente di essa è meno ripida. A parte ciò mi sembra, che come la B, riveli un' azione del campo sull' aria che si trova fra $\mathrm{i}$ poli.

Come spesso accade d'ogni ipotesi suggerita da certi fatti noti, anche l' ipotesi d' una azione del magnetismo tendente a favorire la ionizzazionè dei gas, ha condotto a trovare qualche fatto nuovo. Quelli che ho descritto tendono evidentemente a confermare l' ipotesi assunta. Essa mi parve necessaria per spiegare come un campo possa produrre la scarica quando la differenza di potenziale impiegata non produce nessun passaggio dimostrabile di elettricità prima che il campo esista. $\mathbf{E}$ poichè le nuove esperienze non contraddicono tale ipotesi, sarà utile a mantenerla. 
Essa da sola non vale a rendere conto di tutte le particolarità. E siccome non si può negare la deformazione delle traiettorie degli elettroni per opera del campo, che è la base della spiegazione ordinaria, cosi l'ipotesi della magnetoionizzazione non devesi sostituire, ma aggiungersi all' ordinario modo di spiegare l' azione del campo sulle scariche. Colla magnetoionizzazione si arriva a comprendere come e quando. prenda origine un moto apprezzabile di elettroni in un tubo da scarica per opera del campo; ma bisognerà tener conto del movimento ch'essi assumono sotto l'influenza del campo stesso per prevedere ciò che in seguito avviene, e cioè per sapere, se quella messa in moto di elettroni è destinata ad abortire, oppure ad intensificarsi sino al punto da produrre la scarica durevole.

La questione d' una probabile magnetoionizzazione, oltre che avere una certa importanza per la spiegazione dei fenomeni di scarica nel campo magnetico, ne ha una di gran lunga maggiore dal punto di vista filosofico. Infatti, la constatazione di effetti spiegabili soltanto in base ad una influenza del magnetismo sopra elettroni muoventisi in orbite chiuse entro gli atomi, costituirebbe una validissima conferma sperimentale in favore delle idee, che oggi i fisici vanno formandosi relativamente alle strutture atomiche. 